\title{
Propagation Channel Models for Next-Generation Wireless Communications Systems*
}

Andreas F. MOLISCH ${ }^{\dagger a)}$ and Fredrik TUFVESSON ${ }^{\dagger \dagger \mathrm{b})}$, Nonmembers

SUMMARY As new systems and applications are introduced for nextgeneration wireless systems, the propagation channels in which they operate need to be characterized. This paper discusses propagation channels for four types of next-generation systems: (i) distributed Multiple-Input Multiple-Output (MIMO) and Cooperative MultiPoint (CoMP) systems, which require the characterization of correlation between channels from a mobile station to different base stations or access points; (ii) device-todevice communications, where propagation channels are characterized by strong mobility at both link ends (e.g., in vehicle-to-vehicle communications), and/or significant impact of moving shadowing objects; (iii) fulldimensional MIMO, where antenna arrays extend in both the horizontal and vertical dimension, so that azimuthal and elevation dispersion characteristics of the channel become relevant, and (iv) millimeter wave Wireless Local Area Network (WLAN) and cellular communication systems, where the high carrier frequency leads to a change (compared to microwave communications) concerning which propagation processes are dominant. For each of these areas, we give an overview of measurements and models for key channel properties. A discussion of open issues and possible future research avenues is also provided.

key words: LTE, channel model, CoMP, double-directional, FD-MIMO, device-to-device, vehicle-to-vehicle, $5 G$, mm-wave

\section{Introduction}

Propagation channels determine the fundamental limits of wireless communications, as well as the actual performance of any practical system. Good propagation channel models are thus essential prerequisites for the development and assessment of new systems. In the context of international standards, channel models are furthermore essential to allow fair comparisons of different system proposals. For all of these reasons, development of realistic, yet easy to use, models for wireless propagation channels has been an important and very active research area for many decades.

Channel models should be only as complex as necessary, and thus neglect effects that do not impact the performance of the systems for whose simulation the model is designed. Thus, while physical wireless propagation channels

\section{Manuscript received February 12, 2014.}

Manuscript revised May 19, 2014.

${ }^{\dagger}$ The author is with the Ming-Hsieh Department of Electrical Engineering, University of Southern California, Los Angeles, CA, USA.

${ }^{\dagger \dagger}$ The author is with the Department of Electrical and Information Engineering, Lund University, Lund, Sweden.

*Parts of the work have been funded by Intel Corporate Research, Samsung, an MRI grant of the National Science Foundation, and a grant from the Swedish Foundation for Strategic Research (SSF).

a)E-mail: molisch@usc.edu

b) E-mail: Fredrik.Tufvesson@eit.lth.se

DOI: $10.1587 /$ transcom.E97.B.2022 are independent of the systems that operate in them, channel models do show a dependence. The COST207 channel models [1], designed for assessing second-generation cellular systems like GSM, provide models for the delay dispersion assuming a system bandwidth of $200 \mathrm{kHz}$. The ITU models [2] also concentrate on delay dispersion, though for the wider bandwidth that was relevant for initial $3 \mathrm{G}$ cellular systems. The emergence of multi-antenna techniques in the early $2000 \mathrm{~s}$ motivated the development of a new set of directional channel models, from COST 259 [3], [4], and COST 273 [5] to the 3GPP Spatial Channel Model (SCM) [6], to the ITU/Winner models [7], [8]. At the same time, the emergence of new deployment scenarios, such as microand femtocells, necessitated extending the models for new environments as well. These models served not only for the refinement of the third-generation cellular standards, but also played a critical role in the development of LTE, the current standard for fourth-generation cellular systems [9].

Due to increased demand and new applications, wireless systems continue to evolve. In particular, within the cellular standardization organization 3GPP, a number of new transmission schemes are considered as part of LTEAdvanced. Outside of this cellular development, other organizations such as IEEE 802.11 also continue to develop new systems for either enhanced capabilities or new applications. Some of the most important developments are (i) Cooperative Multipoint (CoMP), (ii) device-to-device communications, (iii) massive MIMO, and (iv) millimeter-wave communications. For all of these applications, new channel models are required.

In CoMP, also known as base station (BS) cooperation or network MIMO, multiple BSs cooperate in order to reduce interference and enhance throughput in each cell, particularly at the cell edge [10], [11]. In its most advanced form (sometimes known as joint transmission, JT), the BSs exchange channel state information, as well as transmit/receive data over the backbone connecting the BSs. The BSs thus essentially form a giant MIMO system, so that there is no intercell interference anymore, and (for the uplink) all transmitted signals are useful signals; in the downlink multi-user MIMO beam forming allows to eliminate interference to each user. Other forms of CoMP rely on coordinating the scheduling such that signals in one cell do not strongly interfere with signals in the neighbor cells that are scheduled on the same time/frequency resource. For all of these applications, it is obvious that signal propagation from/to a mobile station (MS) location to multiple BS loca- 
tions has to be properly modeled. Not only the correlation of the shadowing, but correlations of all other propagation channel parameters, have to be properly described. This is a fundamental generalization of previous channel models (including 3G models), which concentrated on describing the channel from a single BS to one (or more) MSs.

Another emerging transmission scheme is Device-toDevice (D2D) communication, either in an autonomous fashion, or under control of the BS. In the former case, the FlashLinQ system [12] and similar proposals are discussed within 3GPP especially for communication in case of disaster when all infrastructure nodes (BSs) are out of order, and IEEE 802.11p is used for car-to-car communication for safety purposes [13]. In the latter case, it has been shown [14], [15] that coordination of the transmission between devices by means of central knowledge at the BS can also greatly enhance performance. In either case, the propagation channel between devices, which shows very different behavior from BS-to-device channels, has to be accurately modeled. Furthermore, when BS-controlled D2D is implemented, the correlation between the D2D channel and the BS-to-device channel also has to be carefully modeled.

As suggested by [16], increasing the number of BS antenna elements to dozens or hundreds not only increases the spectral efficiency of multi-user communications, but also decreases power consumption and simplifies signal processing. Under the name "massive MIMO", this approach has gained great attention in the past years (see [17], [18] and references therein). In order to accommodate such a large number of antenna elements within a reasonable form factor, three-dimensional antenna structures are required, which exploit not only the azimuthal spread of the multi path components (MPCs) as in traditional cellular MIMO, but also use the elevation spread; this is consequently known as FullDimensional (FD) MIMO [19]. As a consequence, the elevation characteristics of MPCs are in need of careful modeling.

Last, but not least, there is a strong trend to higher frequency bands for data transmission. In particular the mmwave band has much more spectrum available than the microwave bands that have been traditionally used for cellular and Wireless Local Area Network (WLAN) communications. In particular, $60 \mathrm{GHz}$ is the band of choice for multi-GBit/s WLAN systems such as the IEEE 802.11ad standard, while the 28 and $38 \mathrm{GHz}$ bands seem well suited for high-rate cellular communications over distances of up to several hundred meters. Demonstrations and system proposals by operators like NTT DoCoMo, and manufacturers like Samsung, have demonstrated the strong industry interest in such cellular solutions. Since the carrier frequency is an order of magnitude higher than in traditional cellular and WLAN systems, new channel models suitable for this frequency range have to be provided.

The main goal of this paper is to review basic modeling methods and recent results for channel models for these new aspects of next-generation wireless systems. In Sect. 2, we review fundamental channel modeling approaches as used for $3 \mathrm{G}$ systems. Sections 3 to 6 review channel models for CoMP, D2D, FD-MIMO, and mm-wave, respectively. Section 7 summarizes open topics, and Sect. 8 provides conclusions.

\section{Fundamentals of Wireless Channel Models}

This section reviews wireless channel models that are in use for $3 \mathrm{G}$ and early $4 \mathrm{G}$ systems; later sections will then discuss the generalizations currently in development for more modern systems.

Wireless propagation channels are characterized first and foremost by the channel gain, i.e., the ratio of the received power to the transmitted power. Without going into details (see [20] and [21] for those), channel gain averaged over small-scale fading can be written as the sum (on a dB scale) of a (distance-dependent) path gain, and a (stochastic) shadowing term that describes the variations of the strengths of the MPCs. The path gain $G$ is traditionally described by the following equation

$$
G=\frac{E\left\{P_{r}\right\}}{\mathrm{P}_{t}} \sim a\left(\frac{d}{d_{0}}\right)^{-\gamma},
$$

where $P_{t}$ and $P_{r}$ are transmit and receive power, respectively, $d_{0}$ is a reference distance, in the same units as $d$; and $a$ and $\gamma$ are dimensionless model parameters; the expectation $E\{\cdot\}$ is taken over the shadowing and small-scale fading. The probability density function of $S(x)$, the shadowing variations around their mean, is usually modeled as a lognormal distribution, i.e., the logarithm of the variation is a Gaussian-distributed real variable. When considering the spatial scale on which significant changes of the shadowing occur, we consider the autocorrelation function along a path moving towards or away from the TX in the $x$ direction; the correlation between $S$-values for points at $x$ and $x+\Delta x$ can be approximated by a Laplacian function

$$
E\{S(x) S(x+\Delta x)\}=\sigma^{2} \exp \left(-|\Delta x| / X_{c}\right),
$$

where $\mathrm{X}_{c}$ is the correlation distance of shadow fading.

For modern cellular systems, both the delay dispersion and angular dispersion play an important role. In a deterministic description (i.e., for a particular location of transmitter, receiver, and scattering objects), those quantities can be described by the double-directional impulse response [22], which consists of a sum of contributions from the MPCs:

$$
\begin{aligned}
h\left(t, \mathbf{r}_{\mathrm{TX}}, \mathbf{r}_{\mathrm{RX}}, \tau, \Omega, \Psi\right) & =\sum_{l=1}^{L} h_{l}\left(t, \mathbf{r}_{\mathrm{TX}}, \mathbf{r}_{\mathrm{RX}}, \tau, \Omega, \Psi\right) \\
& =\sum_{l=1}^{L} a_{l} \delta\left(\tau-\tau_{\ell}\right) \delta\left(\Omega-\Omega_{\ell}\right) \delta\left(\Psi-\Psi_{\ell}\right),
\end{aligned}
$$

where $\mathbf{r}_{\mathrm{TX}}$ and $\mathbf{r}_{\mathrm{RX}}$ are the locations of the transmitter and receiver, respectively; $\Omega$ and $\Psi$ the direction-of-departure (DoD) and direction-of-arrival (DoA), each of which consists of an azimuth and an elevation component; $\tau$ the delay, 
and $L$ the number of MPCs. The phases of the $a_{l}$ change quickly, while all other parameters, i.e., absolute amplitude $\left|a_{l}\right|$, delay, DoA and DoD vary slowly with the transmit and receive locations (over many wavelengths). It is noteworthy that in this representation, the MPC amplitudes represent the complex gain of the propagation channel only, without any consideration of the antennas.

For multiple-antenna systems, we are also often interested in the impulse response or channel transfer function of the radio channel (i.e., including the antenna characteristics) from each of the $N_{\mathrm{TX}}$ transmit antenna elements to each of the $N_{\mathrm{RX}}$ receive antenna elements. This is given by the impulse response matrix. We denote the transmit and receive element coordinates as $\mathbf{r}_{\mathrm{TX}}^{(1)}, \mathbf{r}_{\mathrm{TX}}^{(2)}, \ldots . . \mathbf{r}_{\mathrm{TX}}^{\left(N_{\mathrm{TX}}\right)}$, and $\mathbf{r}_{\mathrm{RX}}^{(1)}, \mathbf{r}_{\mathrm{RX}}^{(2)}, \ldots . . \mathbf{r}_{\mathrm{RX}}^{\left(N_{\mathrm{RX}}\right)}$, respectively, so that the impulse response from the $i$-th transmit to the $m$-th receive element becomes

$h_{i, m}=h\left(\mathbf{r}_{\mathrm{TX}}^{(i)}, \mathbf{r}_{\mathrm{RX}}^{(m)}\right)=$

$\sum_{\ell} h_{\ell}\left(\mathbf{r}_{\mathrm{TX}}^{(1)}, \mathbf{r}_{\mathrm{RX}}^{(1)}, \tau_{l}, \Omega_{\ell}, \Psi_{\ell}\right) \widetilde{G}_{\mathrm{TX}}\left(\Omega_{\ell}\right) \widetilde{G}_{\mathrm{RX}}\left(\Psi_{\ell}\right)$

$\exp \left(j\left\langle\mathbf{k}\left(\Omega_{\ell}\right),\left(\mathbf{r}_{\mathrm{TX}}^{(i)}-\mathbf{r}_{\mathrm{TX}}^{(1)}\right)\right\rangle\right) \exp \left(j\left\langle\mathbf{k}\left(\Psi_{\ell}\right),\left(\mathbf{r}_{\mathrm{RX}}^{(m)}-\mathbf{r}_{\mathrm{RX}}^{(1)}\right)\right\rangle\right)$,

where $\mathbf{k}$ is the wave vector and $\langle\cdot\rangle$ denotes the inner product; $\widetilde{G}$ is the complex antenna pattern. Note that Eq. (4) implicitly assumes that the DoD (or DoA) at each antenna element is the same, which is well-fulfilled for concentrated antenna arrays, but might not be valid in distributed or physically large arrays, see Sect. 3 .

In order to reflect the variety of channel realizations in nature, stochastic channel descriptions that provide probability density functions (pdf) of the impulse responses, are required. One common form is the "tapped delay line" model, which (in its generalization to the double-directional case) fixes the delay, DoA, and DoD of the MPCs, while allowing the phase and amplitudes to be chosen stochastically according to their pdfs with the common assumption of wide sense stationarity - uncorrelated scattering (WSS-US), i.e., that the statistics of the fading do not change with time, and fading of each MPC is uncorrelated. Such an approach underlies the 3GPP SCM as well as the ITU/Winner models. An alternative approach is the Geometry-based Stochastic Channel Model (GSCM) [23], where locations of scatterers or interacting objects (IOs) are defined according to a given probability density function, and the characteristics of the MPCs are finally obtained through a simple ray tracing procedure that allows only single-interaction processes or (as in the COST 273 and COST 2100 models) double interactions with so-called "twin clusters" [5]. In many cases, tapped delay line and geometry-based approaches are combined, such that the "locations" (i.e., delays, DoAs, and DoDs) of the taps are obtained from geometric considerations.

Another important concept in modern channel modeling is clustering. Measurement results show that in many environments, MPCs arrive in clusters, i.e., groups with similar characteristics [24]-[26]. This effect arises, e.g., because the MPCs are created by the interaction of the transmit sig- nal with objects such as a group of high-rise buildings or mountains, or from waves undergoing similar waveguiding processes in a corridor or street canyon. Clusters can be extracted from double-directional impulse responses through visual inspection [26], [27] or automated processing [28].

The concept of clusters is useful because the parameters of a cluster do not change even when an MS moves over a larger area. To give an example, the PDP (power delay profile, i.e., expected magnitude of the squared impulse response, [20]) of a single cluster often can be modeled as an exponential function. When the channel contributions come from three clusters, the PDP of the overall channel consists of three exponentials. When the MS moves over large areas, the position of the exponentials relative to each other changes, but the shapes of the cluster PDPs remain unchanged. Furthermore, a cluster shape function (squared magnitude of the double-directional impulse response of the cluster, averaged over the small-scale fading) can often be decomposed, e.g., [4]

$P\left(\tau, \theta, \varphi, \theta^{\prime}, \varphi^{\prime}\right)=P_{\tau}(\tau) P_{\theta}(\theta) P_{\varphi}(\varphi) P_{\theta^{\prime}}\left(\theta^{\prime}, \tau\right) P_{\varphi^{\prime}}\left(\varphi^{\prime}, \tau\right)$,

where $\theta, \varphi$ are the elevation and azimuth at the BS (and analogously for $\theta^{\prime}, \varphi^{\prime}$ at the MS). It is common to assume that the PDP is a single-exponential function, while the angular power spectra are Laplacian functions. The second central moments of these functions (delay spread, azimuth spreads, elevation spreads) are then commonly used for the characterization of the environments.

In order to model the appearance and disappearance of clusters (except the cluster of MPCs scattered near the MS, which is always present), we define "visibility regions" in a coverage area so that if the MS is in such a region, the cluster is active, i.e., the MPCs belonging to that cluster contribute to the double-directional impulse response; otherwise they are not, see Fig. 1. The visibility regions are placed at random in the cell area, with the pdf of the visibility region centers being a parameter of the model. It is furthermore common to define a "transition function" that ensures that when an MS enters a visibility region, the MPC cluster does not activate all of a sudden (which would lead to a discontinuity in power). An alternative to the visibility region is a birth/death process, where clusters are turned on or off at random times.

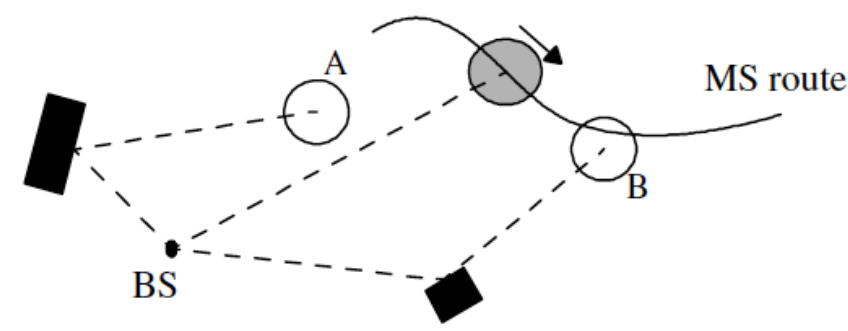

Fig. 1 Concept of visibility region: clusters become visible when MS moves into associated visibility region. From [29]. 


\section{Multi-Link Channel Models}

While in third-generation systems MIMO was mainly used to enhance the capacity of each link separately, nextgeneration systems make more extensive use of multi-link coordination or -cooperation. Multi-user MIMO has already been introduced in IEEE $802.11 \mathrm{ac}$, and will gain an increasingly important role for cellular systems as well. Channel modeling for multi-user MIMO does not, however, require any new techniques, since the existing spatial channel models (like 3GPP-SCM, ITU/Winner, etc.) already allow for dropping multiple users within one cell, and/or (equivalently) an MS moving through the cell. However, major challenges arise in modeling the links from a single MS to multiple BSs, which is required in particular for cooperative multipoint systems (CoMP) and distributed antenna systems. In those cases, as well as for multi-link channel modeling in general, it is essential to capture correlation of the statistics between the considered links. It cannot be assumed that the large-scale parameters such as rms delay spread, angular spread, and large-scale fading are independent just because the nodes are highly separated. Rather there tends to be some degree of correlation for these large-scale parameters.

Much of the early work in this area has concentrated on the correlation of the shadowing between BSs (a rudimentary model for this effect was even included in the 3GPP SCM). Besides this, also other large-scale parameters such as angular spread, delay spread, etc., can show correlation between the links. A number of measurement campaigns have been devoted to identifying these correlations. In [30] correlation between large-scale parameters for a macro cell scenario at $2.6 \mathrm{GHz}$ was analyzed and it was found that the parameters of different links can be correlated even if the BSs are far away from each other. When both BSs were in the same direction compared to the movement the largescale parameters of the different links had a tendency to be positively correlated, but slightly negatively correlated when the BSs were located in different directions compared to the movement of the mobile terminal. The spatial structure of the same dataset was studied in [31] and it was found that the average capacity could be increased by $53 \%$ by cooperative BSs. Correlation properties between BSs were also analyzed in [32], showing measured correlation between different links that actually was somewhat smaller than that predicted by the 3GPP SCM. In [33] measured values of the cross correlation properties in a dual link urban scenario at $3.7 \mathrm{GHz}$ were presented, while in [34] cross correlation properties of 4 coherent links in a sub-urban scenario at $2.6 \mathrm{GHz}$ are presented. Cross correlation coefficients between large scale parameters reach values up to \pm 0.8 and are typically modeled by a joint, often Gaussian, correlated distribution.

For conventional single link MIMO, the correlation between antennas is usually of high interest. When the nodes are widely separated, the correlation of the small-scale fad- ing between single antennas at different nodes can under normal circumstances be neglected, but of course correlation among antennas at the same node is still important. Similarly the spatial structure of separate MIMO links can be correlated. Such a spatial separation of different MIMO links in an office scenario was analyzed and modeled in [35]. It was found that the spatial separation (from a signal processing view) sometimes is large and sometimes is low, but it can not easily be connected to the direction to, and distance between, nodes. In addition, the power imbalances when the nodes are separated is another important factor to model as this will affect the SNRs of the considered links significantly. These power imbalances can easily be calculated by standard pathloss expressions, possibly together with an appropriate correlated large-scale fading model.

In order to simulate correlation properties in multilink scenarios, Poutanen et al. [36] developed the concept of common clusters (sometimes also referred to as joint or shared clusters). The basic idea behind common clusters is that users sometimes use the same clusters and hence they partially use the same propagation paths in the simulations, with the result of correlated MIMO links. The common cluster concept is adopted in the COST 2100 model [37] for multilink simulations. In the model, several visibility regions can be associated with each cluster, so if two users happen to be in visibility regions associated with the same cluster this cluster will be a common cluster, and hence there will be some correlation between the corresponding links. Similarly, each BS has many visibility regions distributed in the simulation area, and this distribution defines the number of BSs that can be used for cooperative communication with a particular user. The different types of common clusters are described in Fig. 2. An example for the relative importance of common clusters (i.e., how much power is carried) can be found in Fig. 3.

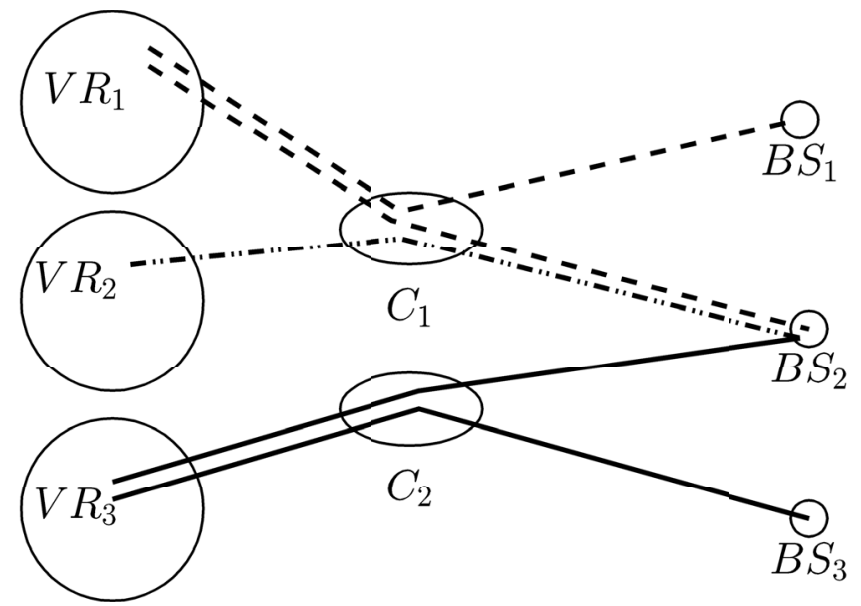

Fig. 2 Different kinds of common clusters. The clusters $C_{1}$ and $C_{2}$ are associated to three BSs and three users in the visibility regions. The lines define the visibility of the different users to the BSs. From [37]. 


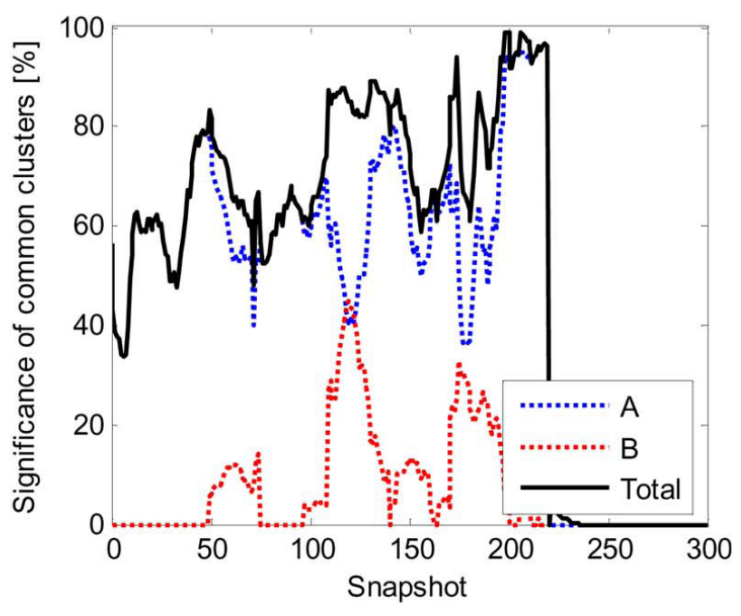

Fig. 3 Significance of common clusters for connections from MS to two BSs in an indoor environment. From [36].

\section{Device-to-Device Models}

Device-to-device (D2D) radio channels have fundamentally different properties compared to those of conventional cellular channels. The main reason for this is that most often both the receive antenna and the transmit antenna are located at low heights, and hence there is more interaction with objects in the close neighborhood of the devices. Due to the fact that the two devices are at approximately the same height, the propagation effects are significantly different from traditional device-to-infrastructure (D2I) links. This difference is especially pronounced for outdoor links, where a BS would be high above ground (typically $10 \mathrm{~m}$ for microcell, and up to $100 \mathrm{~m}$ for macrocells), while all devices are at street level. Consequently, over-the-rooftop propagation is not a viable mechanism, and even street canyon propagation is more strongly affected by shadowing objects such as cars and trucks. The most frequently occurring application of outdoor D2D systems is vehicle-to-vehicle (V2V) communications, though other applications are also thinkable. In indoor situations, the difference between D2I and D2D propagation mechanisms is less pronounced, and the range of validity for many indoor channel models includes the D2D case.

Pathloss: A first impact of the different propagation conditions is the pathloss model. For outdoor situations, the pathloss exponents are generally in the range 1.6-2 when the two devices communicating with each other are in the same street (in urban, suburban, or rural environments) or on the same highway [38]. When the two devices are on orthogonal streets, the model of [39], based on extensive measurements in and around Munich, Germany, proposes

$$
\begin{aligned}
& P L\left(d_{r}, d_{t}, w_{r}, x_{t}, i_{s}\right)=C+i_{s} L_{S U} \\
& + \begin{cases}10 \log _{10}\left(\left(\frac{d_{t}^{E_{T}}}{\left(x_{t} w_{r}\right)^{E_{S}}} \frac{4 \pi d_{r}}{\lambda}\right)^{E_{L}}\right), & \text { if } d_{r} \leq d_{b} \\
10 \log _{10}\left(\left(\frac{d_{t}^{E_{T}}}{\left(x_{t} w_{r}\right)^{E_{S}}} \frac{4 \pi d_{r}^{2}}{\lambda d_{b}}\right)^{E_{L}}\right), & \text { if } d_{r}>d_{b}\end{cases}
\end{aligned}
$$

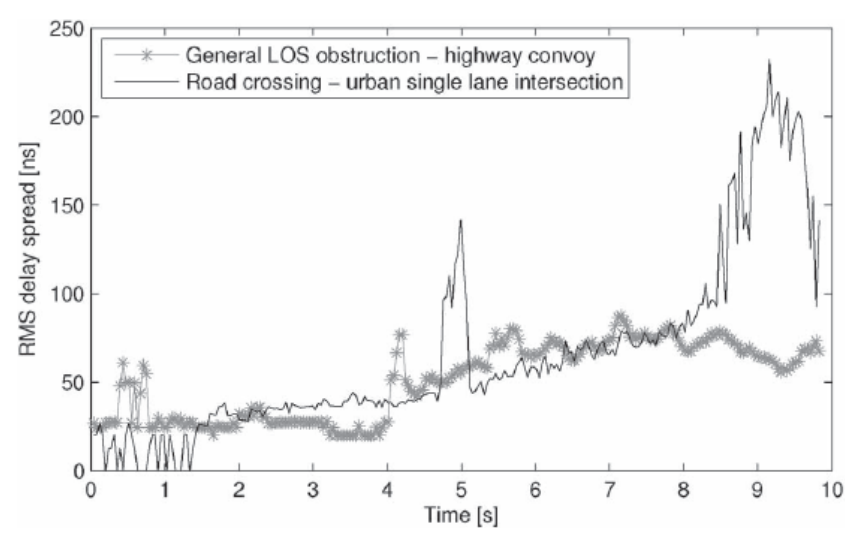

Fig. 4 Evolution of rms delay spread as car is moving in two different environments. From [45].

where $d_{t}$ and $d_{r}$ denote the distance of the TX and RX to the intersection center, respectively, $w_{r}$ is the width of the RX street, and $x_{t}$ is the distance of the TX to the wall. In the model $C=3.75 \mathrm{~dB}$ is the so-called curve shift, $L_{S U}=2.94 \mathrm{~dB}$ is the sub-urban loss, $i_{s}=0$ is the urban loss factor, $i_{s}=1$ the sub-urban loss factor, $E_{L}=2.69$ is the loss exponent, $E_{S}=0.81$ is the street exponent, $E_{T}=0.957$ is the TX distance exponent, and finally $d_{b}=180 \mathrm{~m}$ is the break even distance. The model was later validated by independent measurement data by Abbas et al. [40] with good agreement, but also with the suggestion to include a random offset parameter to reflect variations between different street intersections; random variations were also suggested in [41] based on the comparison of ray tracing results with the model of [42].

Delay Dispersion: As far as the delay dispersion is concerned, the rms delay spread (second central moment of the power delay profile) for $\mathrm{V} 2 \mathrm{~V}$ environments varies as a function of location, and can be modeled as a random variable (log normally distributed in most cases). Mean rms delay spreads are on the order of 100-200 ns for rural and suburban environments, and up to $400 \mathrm{~ns}$ for urban environments. Detailed reviews of the various measurement results for both pathloss and delay dispersion for various environments can be found in [43], [44]. Delay spreads in safety related scenarios for $\mathrm{V} 2 \mathrm{~V}$ communication are, e.g., reported in [45], see also Fig. 4. High rms delay spreads are observed when the line-of-sight component is blocked and there are large reflecting objects close to the road. Mean rms delay spreads of $170 \mathrm{~ns}$ are reported for longer periods, but there are also extreme values of the rms delay spread just below $1 \mu \mathrm{s}$.

Temporal Variations: There are two distinct groups of D2D channels, depending on the dynamics of the nodes, i.e. if the devices themselves are moving or not. In the first case, devices at both link ends can move, sometimes very fast. In addition scatterers and shadowing objects can also move. This is, e.g., the case for V2V channels, for which extensive research has shown that the channel statistics typically change over time and hence the conventional assumption about WSS-US (see Sect. 2) is only fulfilled for 


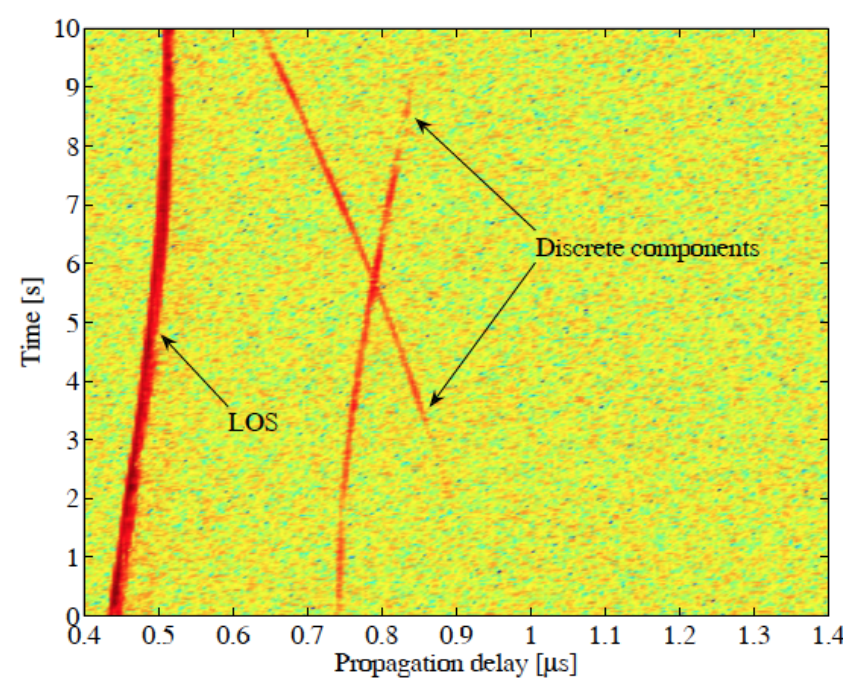

Fig. 5 Example plot of a time-varying power delay profile of V2V communications on a highway. From [48].

rather short time intervals, and moderate frequency intervals. Figure 5 shows an example of a PDP; it is obvious that the relative delay between the MPCs created by the line-ofsight (LOS) and various discrete components changes significantly over time, thus limiting the duration over which WSS is valid. In [46] a stationarity bandwidth of around $50 \mathrm{MHz}$ and a stationarity time corresponding to a movement of 10-40 m were suggested, whereas [47] reported somewhat larger values for the stationarity bandwidth, but smaller values for the stationarity time. Delay spreads and Doppler spreads in safety related scenarios for V2V communication are, e.g., reported in [45]. High Doppler spreads typically arise in drive-by situations, with mean rms Doppler spreads of $300 \mathrm{~Hz}$ are reported over a longer time, but with extreme values of just below $1000 \mathrm{~Hz}$.

In order to handle the non-stationarities from a channel modeling perspective, the most straightforward solution is often to use a GSCM [49], where the non-stationarities are automatically taken care of and modeled by the randomly placed scatterers in the environment. Examples of GSCMs for V2V scenarios can be found in [48]-[50], see Fig. 6. Another approach is based on tapped delay lines, such that the location (delay) of the taps is either adjusted continuously, or a birth/death process of the taps is implemented; the latter method is used, e.g., in the IEEE 802.11p channel model [51].

In static or nomadic scenarios, the two nodes do not change with respect to each other. This occurs, e.g., in machine-to-machine communications (static nodes) or peerto-peer (e.g., WiFi Direct) links between laptops (nomadic scenarios). In that case the Doppler spectrum of the channel is determined by moving objects in the surroundings. Typically the Doppler spread is low as many of the dominant scatterers are static as well and hence the coherence time of the channel can be quite large [52]. However, one important aspect that should not be forgotten is the shadowing or

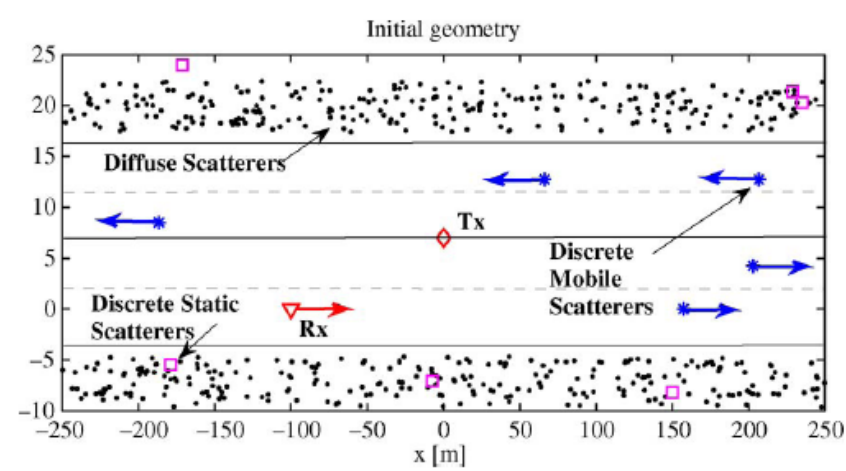

Fig. 6 Principle of geometry-based stochastic channel model for V2V communications. From [43].

blocking of the radio signal by other objects as the coherence time of this process can be very large as well.

\section{3-D Channel Modeling}

Most of the directional channel models in the literature, including the 3GPP-SCM models, concentrate on the directional characteristics in the azimuthal domain. This is related to the fact that capacity improvements by multipleantenna systems are greatest when the angular spread is large, which usually occurs in the azimuth domain. In the elevation plane, the angles of the MPCs as seen from the BS are usually restricted to a fairly narrow range, which has traditionally been taken into account by using BS antennas with a narrow, but non-adaptive beam width. The emergence of massive MIMO systems [17] has however increased the interest in using the elevation domain as well, since arrays with dozens or hundreds of antenna elements can achieve a reasonable form factor only when spanning at least two dimensions [19], [53]. In [54] an extension of the COST 2100 model for massive MIMO systems is proposed. However, the best configuration in terms of antennas in the horizontal and vertical domain is still an open issue. Thus, the elevation components of $\Omega$ and $\Psi$ in Eqs. (3), (4) become highly important.

Measurements of the elevation characteristics of propagation channels fall into two categories: elevation spectrum (i) at the BS, and (ii) at the MS (depending on whether we consider uplink or downlink, direction at the BS might be DoA or DoD, respectively). Historically, elevation spectra at the MS were measured first, as they showed greater spreads and provided valuable insights into propagation mechanisms. MPCs arriving at the MS via over-the-rooftop propagation in urban environments tend to have high elevation angles ${ }^{\dagger}$ associated with the last diffraction from the rooftop to the street in which the MS is located; while MPCs that are waveguided in street canyons have lower elevation [55], [56]. Extensive measurements of [57] showed that an (asymmetrial) double-exponential function provides a good fit for the elevation power spectrum measured at the MSs,

†hile different definitions of elevation exist, in this paper we define it as the angle measured from the horizontal plane. 

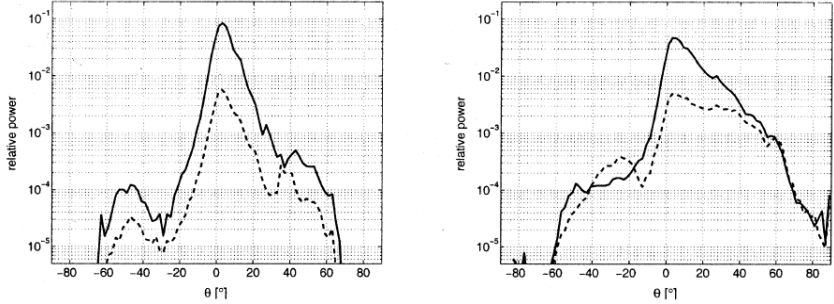

Fig. 7 Elevation spectra for urban microcell (left) and macrocell (right) at the MS. From [57]; with permission by IEEE.

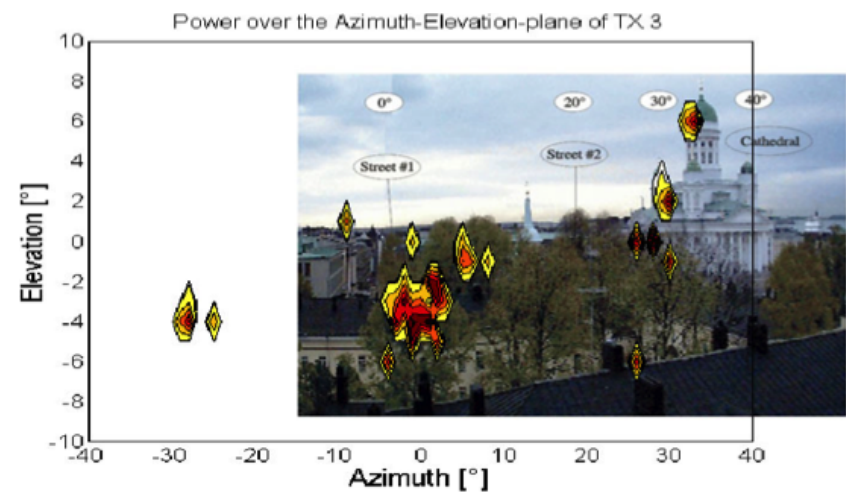

Fig. 8 Propagation processes leading to different elevations. From [61].

see Fig. 7. Elevation spreads measured in that reference were typically less than $10-15$ degrees, while other references measured somewhat larger values in both outdoor [58] and indoor [59] environments; the differences are most likely due to the different building structures in the measured cities.

Elevation spreads at the BS are considerably smaller. References [60], [61] evaluated a number of measurements with rectangular antenna arrays at the BS. They found that over-the-rooftop propagation, wave guiding in street canyons, and reflections off high-rise buildings and dominant scatterers provide different contributions to the elevation spectrum, see Fig. 8. Clustering of the waves can be observed. The overall angular power spectrum in the elevation domain can thus be modeled as a sum of those contributions; see also [62].

Initial theoretical investigations of FD-MIMO systems had conjectured that the mean elevation angle would be determined by the line-of-sight connection between BS and MS, even if the LOS is blocked. However, measurements (e.g., [63]) have shown that the mean angle is closer to the horizontal plane at both the MS and the BS, in particular for NLOS situations. The elevation spread also has a distance dependence (generally smaller spread at larger distances) [64]. Further ray tracing studies [65] investigated the impact of the BS height on the elevation spreads at BS and MS. The BS spread showed only minor variations (only in the far tails of the elevation spread). [57] also found that the MS elevation spread increases as the BS height increases. Figure 9 also shows that the elevation spread in outdoor-

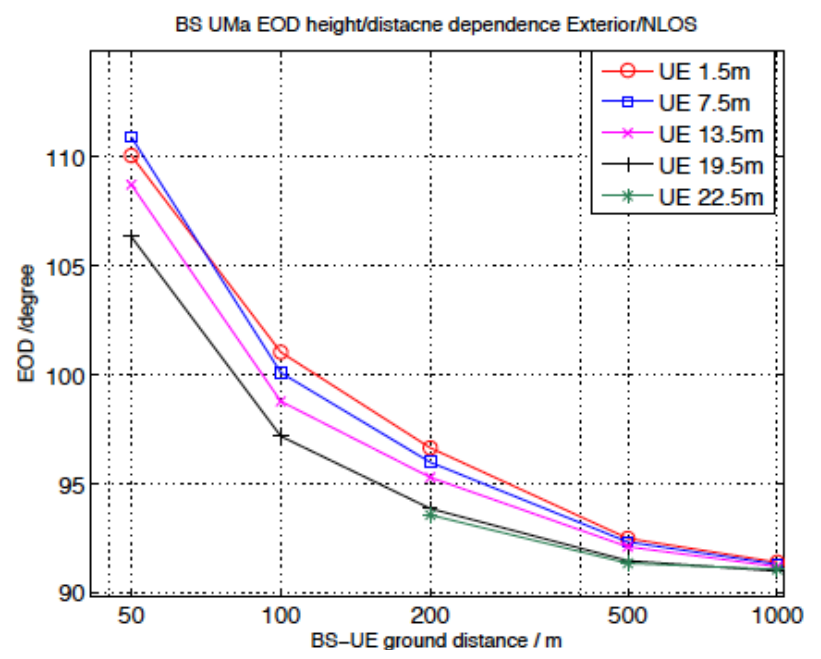

Fig. 9 Distance dependence of the elevation spread for different MS(UE) heights. Note that EoD in this graph is "elevation +90 degree". From [68].

to-indoor scenarios tends to be lower when the MS is on a higher floor.

For system simulations, a number of extensions of the ITU/Winner model (which itself is an extension of the 3GPP-SCM model) have been proposed. Since the ITU/Winner model is based on clusters, we have to distinguish between cluster spreads and composite spreads. Also noteworthy is the nomenclature of these models: the ITU/Winner models implicitly assume a downlink, so that "departure" really means "BS" and "arrival" means "MS" (naturally the spread at a particular station should be independent of whether it operates in transmit or receive mode). The elevation spread is modeled as a log normally distributed variable whose mean and variance are parameterized for the different environments; consistent with the modeling of the azimuthal spread. Studies based on measurements and ray tracing [64], [66], [67] suggested elevation spreads and mean elevations for indoor, hotspot, outdoorto-indoor, urban microcell and urban macrocell; elevation spreads at the BS are typically less than 10 degrees.

Over the past year, a subgroup within 3GPP has been developing a channel model for three-dimensional arrays. The structure of the model is similar to the ITU/Winner model. The parameterization is still in progress; it is based on three types of results: (i) ray tracing results in "virtual" cities with a regular street grid and buildings chosen from a prescribed probability density function; (ii) ray tracing based on three-dimensional maps of actual cities, and (iii) several measurement campaigns. Topics of particular interest are the dependence of the mean elevation and elevation spread as a function of the distance. Considered environments include urban macrocells and urban microcells, both for the case that the MS is outdoors, and that it is indoors. In the latter case, the dependence of the elevation characteristics on the height of the MS (i.e., which floor of the building it is on) deserves particular attention. The final report of the subgroup is expected for summer/fall 2014. 


\section{Millimeter-Wave Propagation}

While the amount of available spectrum in the microwave range is very limited, the mm-wave range offers a large amount of hitherto unused spectrum. In the $60 \mathrm{GHz}$ band, up to $7 \mathrm{GHz}$ bandwidth (depending on the spectrum regulations in the different regions) have been made available for unlicensed wireless systems. For the 28 and $38 \mathrm{GHz}$ bands, about $1 \mathrm{GHz}$ of bandwidth is available as well. A major reason why this spectrum has been unused for a long time is the cost of mass-producing chips for mm-wave frequencies; for a long time Gallium Arsenide and other costly materials had to be used. However, recent years have seen the emergence of CMOS technology that is capable of handling these high frequencies. Thus, low-cost production for massmarket consumer application has become feasible.

Another long-standing obstacle for the realization of mm-wave systems has been the high free-space pathloss. However, by using high-gain antennas, this pathloss may be compensated. Again, recent years have seen progress in the realization of integrated antenna arrays for these frequency ranges that allow to achieve reasonable coverage. As a consequence, cellular outdoor communication (over a range of some $200 \mathrm{~m}$ ) seems feasible, in addition to short-range indoor applications for consumer electronics, and directional microwave links for backhaul. For all of these applications, new channel models are required.

A first step lies in the establishment of pathloss models for $60 \mathrm{GHz}$. A number of factors become essential that are not of common concern in the microwave region. Firstly, atmospheric conditions can impact the attenuation. Oxygen attenuation is on the order of $10 \mathrm{~dB} / \mathrm{km}$. This is a significant problem for long-distance directional links; however, it hardly plays a role for indoor or cellular links that are typically no longer than $200 \mathrm{~m}$ [69], [70]. Also rainfall leads only to an attenuation of about $1.5 \mathrm{~dB}$ over a $200 \mathrm{~m}$ link distance [71], though extreme weather can lead to significantly larger attenuation [72], [73]. Foliage can also be a significant source of attenuation [72], [74], [75].

For multi path propagation, we observe a number of key effects:

- transmission through walls is very low, so that outdoorto-indoor penetration, as well transmission from one room to the next, are greatly attenuated,

- reflection coefficients for reflection from smooth walls and other objects such as lampposts are large (often larger than 0.8 ), giving rise to very efficient propagation paths from transmitter to receiver [76], though surfaces such as brick have a much lower specular reflection coefficient in the $\mathrm{mm}$-wave range than at lower frequencies,

- diffraction is a very inefficient mechanism at high frequencies.

From the above, it follows that (i) outdoor-to-indoor penetration is very small; (ii) outdoor propagation is mostly de-

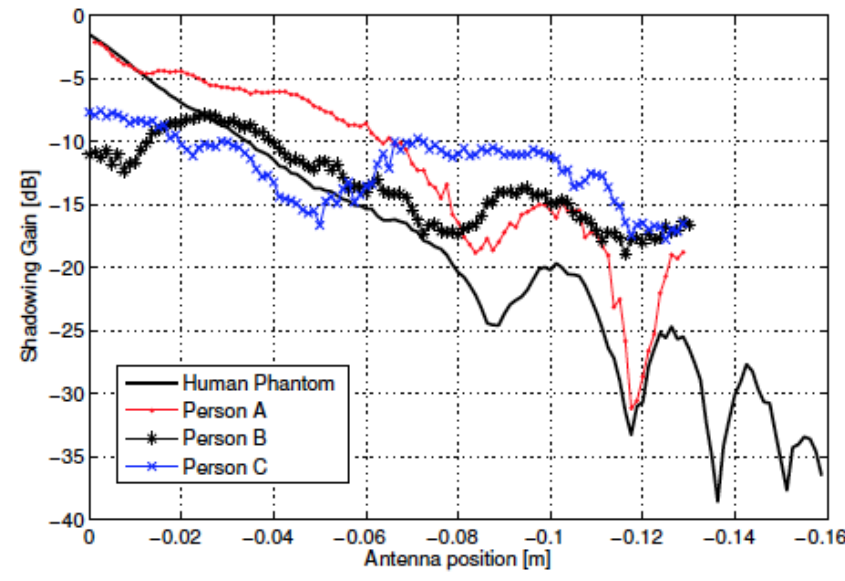

Fig. 10 Body shadowing loss at $60 \mathrm{GHz}$ for three different persons From [77].

termined by the existence of effective reflection paths, while diffraction is highly inefficient; (iii) for outdoor environments, the type of building surfaces (e.g., glass versus rough brick) plays a major role; (iv) in indoor environments, links are usually limited to stations within one room, as penetration from one room to the next is negligible. A further very important effect for $60 \mathrm{GHz}$ systems is shadowing by human bodies. Shadowing losses are on the order of 10-20 dB, where variations between different shadowing persons can be significant [77]. Figure 10 shows the shadowing loss as a probe antenna is moved from a LOS position to a placement behind the shadowing body.

These propagation characteristics cause the channel to be sparse, i.e., there are only a few significant MPCs. This has a very important impact on the double-directional impulse response, as described below, but also on the received power that is obtained under combining diversity at the receiver. [69] defines an "effective" pathloss exponent, which describes the distance dependence of the power at the output of a combiner with $Q$ branches. With $Q=3$, this exponent can be on the order of 3 even in NLOS situations, and thus allow coverage of $200-300 \mathrm{~m}$ cells with NLOS.

Delay spreads play a very important role for $\mathrm{mm}$-wave systems since they are to be operated at extremely high data rates and thus short symbol (or chip) durations. For indoor environments, typical delay spreads between 15 and $60 \mathrm{~ns}$ have been observed [78], while for outdoor cellular environments values are usually below $100 \mathrm{~ns}$ [79]. The delay spreads in systems with highly directive (13-25 dB gain) antennas are often on the order of $20 \mathrm{~ns}$ or less [76], [80], [81]. The delay spread tends to increase with decreasing BS height, which is a behavior similar to other frequency ranges.

Since $60 \mathrm{GHz}$ systems require high-gain antennas, which in a mobile system are implemented by means of adaptive arrays, the directional characteristics of the propagation channel are of great importance. $60 \mathrm{GHz}$ models usually follow the standard generalization of the SalehValenzuela model. This is true for two standardized $60 \mathrm{GHz}$ 


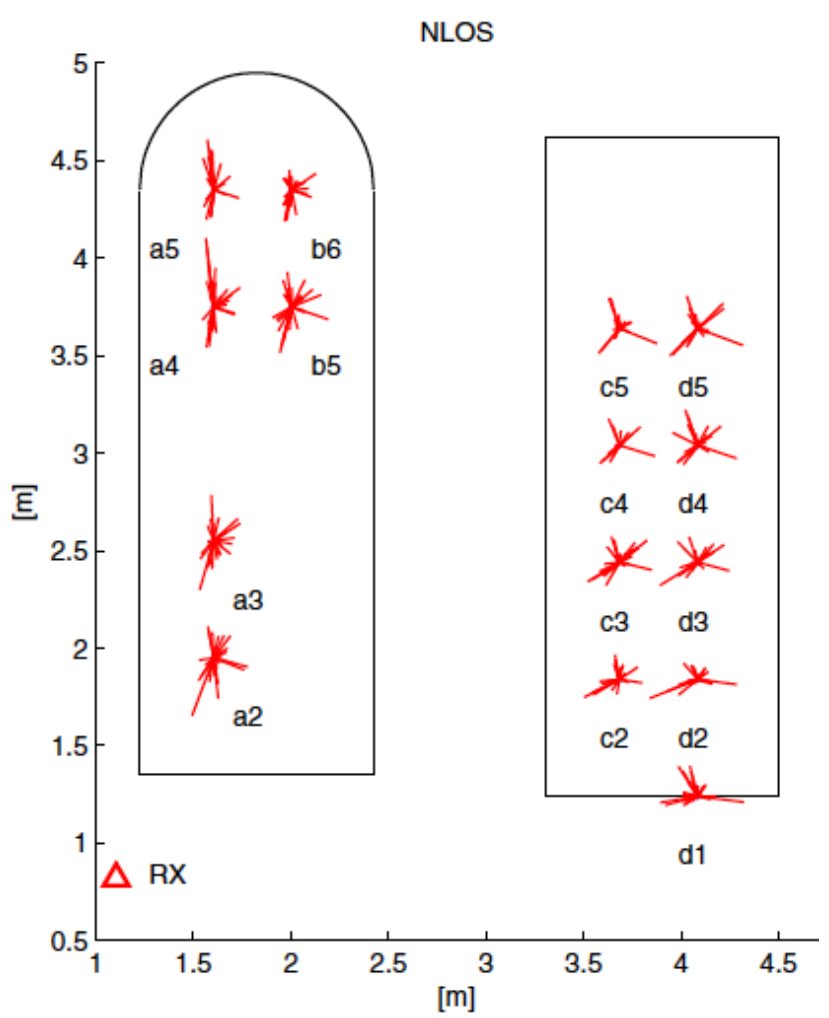

Fig. 11 Power angular spectra for in-room $60 \mathrm{GHz}$ system. From [86].

models, the IEEE 802.15.3c channel model [82], [83] and the IEEE 802.11ad model [84]. Those provide models for residential, office, library, desktop, and kiosk environments. The number of clusters is between 4 and 12; Rice factors are around $10 \mathrm{~dB}$. [85] provides double-directional measurements, and finds somewhat larger angular spreads than the IEEE 802.15.3c model (see Fig. 11 for an example). For outdoor environments, [76] gives example directional characteristics, but statistical models have not yet been extracted.

\section{Open Topics}

While a lot of work has been done, there are a large number of open issues for channel models for next-generation wireless systems. Firstly, next-generation systems will not exclusively consist of the four types of systems discussed in this paper $^{\dagger}$, but even for the systems discussed here, considerable effort will be required in order to ensure understanding of propagation channels that will enable robust and efficient system design.

A first challenge lies in the performance of large-scale channel measurements. As channel measurements and their evaluations have become more and more complicated, the difficulties in performing channel measurements have vastly increased. For double-directional channel measurements,

Other areas of interest include, but are not limited to, bodyarea networks, femto-cells, sensor networks, etc. As these are out of the scope of the current paper, we refrain from a further discussion. switched channel sounders have become the standard measurement approach, where (at the transmitter) a sounding signal created by a single waveform generator is sequentially applied to the different transmit antenna elements, and the received signal is similarly sequentially measured at the different receive antenna elements. However, this measurement principle faces limitations for several of the channel types discussed in this paper: (i) For distributed MIMO systems/CoMP, signals have to be transmitted, via cables, from the waveform generator to the antennas that can be several hundred meter or more away. This can lead to large signal attenuation. Electro-optical conversion and transmission via optical fiber can alleviate the attenuation problem, but proper calibration and temporal stability remains a challenge. (ii) For D2D communication with fast-moving links, channel identifiability becomes difficult, as the channel might change significantly during the time it takes for the sounder to switch through all transmit and receive antenna elements. For massive MIMO systems, a similar challenge occurs - while the temporal variability might be smaller, the number of antenna elements that has to be switched through is much larger. Finally, it is difficult to obtain switches that are suitable for mm-wave frequencies, thus often necessitating different measurement principles such as mechanically moved or rotated antennas. Last, but not least, the demand for measurements with larger and larger bandwidth increases the requirements for accuracy of frequency references, and also increases the sheer amount of data that is collected (it is not unusual for a measurement campaign to collect several Terabyte of data). As measurements remain the essential basis for all channel models, further improvements in measurement equipment will remain a key area of research. The gaps in our understanding of specific models are also mostly caused by a lack of measurements, which in turn is related to the difficulties and effort of performing large-scale campaigns. It must be emphasized that a typical measurement campaign (lasting one week, and providing data for one or two typical environments and applications) requires several months of preparation and between half a year and a year of evaluation.

For multi-link models, very few measurements provide joint double-directional evaluations at multiple TX/RX locations. This makes it difficult to derive critical parameters of multi-link models, such as correlations between angular spreads, identification of joint clusters, etc. The community is only at the very early stages of developing such a model. This has important impact on system design. Evaluations of CoMP schemes within 3GPP have provided mostly disappointing results for the capacity improvement; yet it is not clear at this time to what degree these results are a consequence of the (oversimplified) channel models applied in these simulations.

For V2V communications systems, pathloss and delay spread in different environments (such as urban, rural, etc.) have been measured in a number of different cities and countries. However, the vehicle types and antenna mounting positions used in these measurements have been very limited. 
Measurements between trucks and/or buses are mostly missing. Also, the impact of vehicles between the transmitter and receiver is mostly unknown. How would the shadowing be impacted by a big tractor/trailer located between the transmitter and receiver? How do cars parked on the side of the road impact the performance? Last but not least, what are the directional characteristics of the channels at the cars (related to the question of what performance gain could be achieved by multiple-antenna systems)? All these questions will have to be resolved in order to assess the feasible reliability of V2V systems, especially for safety-critical applications such as collision warnings.

For elevation characteristics, the biggest gaps in existing data sets are related to outdoor-to-indoor links. Clearly, the height of the MS (i.e., on which floor it is located) influences the elevation characteristics, but up to now investigations have been limited mostly to ray tracing simulations. Even then, the impact of the indoor floor plan, and the horizontal location of the MS within the building, have not yet been investigated extensively (though see [68]).

For mm-wave channels, pathloss and delay dispersion in indoor channels have been investigated quite extensively. Directional characterization generally faces larger problems, due to the difficulty of measuring it effectively (see above). While the IEEE 802.15.3c and IEEE 802.11 ad models provide some directional information, additional measurements for verification in a variety of environments would be desirable. More importantly, directional characterizations in outdoor environments are almost completely lacking (though note the recent work of Rappaport et al.). Furthermore, polarization properties and the characterization of temporal variations in mm-wave channels, especially due to the people acting as moving shadowing objects disrupting the LOS, need further investigations.

In the area of generic channel modeling approaches, the key challenges lie in multi-link channel models. The correlation of the properties between the different links is critically important, but also very difficult to model. The different links see some joint clusters, and some disjoint clusters. While the COST 2100 channel model has laid extremely important groundwork, further improvements are required. In particular, a generalization that is capable of dealing with D2D communications needs to be developed. Even for the seemingly simple case of 3 links out of which 2 are moving gives rise to extremely challenging correlated shadowing processes (not just coefficients) [41].

In addition to the pure channel measurement and modeling aspect, the question of how the various modeling approaches and parameterizations influence system performance, and how systems can be designed in order to best operate in those channels, needs to be investigated. Understanding propagation channels is a main inspiration for better and more efficient system design (see, as one typical example, the interplay between mm-wave channel modeling and massive MIMO system design in [87]).

\section{Conclusions}

This paper discussed propagation channels for four scenarios that will be of great importance for next-generation wireless systems: multi-link, device-to-device, FD-MIMO, and $\mathrm{mm}$-wave. After first reviewing basic propagation channel characterization methods, we then reviewed the main measurement results available in the literature, and the models that have been derived from them. For some of the scenarios, such as multi-link, FD-MIMO, and D2D, international standardization activities are on-going. Despite the important and extensive work that has been done up to now, a lot of open questions remain, and extensive measurement and modeling activities will be required to gain an understanding and quantification of channels that is sufficient for robust and efficient system design.

\section{References}

[1] M. Failli, ed., COST207 - Digital Land Mobile Radio Communications - Final Report. Commission of the European Communities, 1989.

[2] ITU, "Recommendation m. 1225," Guidelines for evaluation of radio transmission technologies (RTT) for IMT-2000, 1997.

[3] A.F. Molisch, H. Asplund, R. Heddergott, M. Steinbauer, and T. Zwick, "The COST259 directional channel model part I: Overview and methodology," IEEE Trans. Wireless Commun., vol.5, pp.3421-3433, Dec. 2006.

[4] H. Asplund, A.A. Glazunov, A.F. Molisch, K.I. Pedersen, and M. Steinbauer, "The COST259 directional channel model part II: Macrocells," IEEE Trans. Wireless Commun., vol.5, pp.3434-3450, Dec. 2006.

[5] H. Hofstetter, A.F. Molisch, and N. Czink, "A twin-cluster MIMO channel model," First European Conference on Antennas and Propagation, 2006. EuCAP 2006, pp.1-8, 2006.

[6] G. Calcev, D. Chizhik, B. Goransson, S. Howard, H. Huang, A. Kogiantis, A.F. Molisch, A.L. Moustakas, D. Reed, and H. Xu, "A wideband spatial channel model for system-wide simulations," IEEE Trans. Veh. Technol., vol.56, no.2, pp.389-403, 2007.

[7] WINNER II, IST, "D1. 1.2WINNER II Channel Models Part I Channel Models," Sept. 2007.

[8] ITU-R Report M.2135, "Guidelines for evaluation of radio interface technologies for imt-advanced," 2008. http://www.itu.int/publ/RREP-M.2135-2008/en

[9] E. Dahlman, S. Parkvall, and J. Skold, 4G: LTE/LTE-Advanced for Mobile Broadband: LTE/LTE-Advanced for Mobile Broadband, Academic Press, 2011

[10] A.F. Molisch, M.V. Clark, H. Dai, M.Z. Win, and J.H. Winters, "Method and apparatus for reducing interference in multiple-inputmultiple-output (MIMO) systems," March 22 filed 2002, issued 2011. US Patent 7,912,014.

[11] P. Marsch and G.P. Fettweis, Coordinated Multi-Point in Mobile Communications: From theory to practice, Cambridge University Press, 2011.

[12] X. Wu, S. Tavildar, S. Shakkottai, T. Richardson, J. Li, R. Laroia, and A. Jovicic, "Flashlinq: A synchronous distributed scheduler for peer-to-peer ad hoc networks," 48th Annual Allerton Conference on Communication, Control, and Computing (Allerton), 2010, pp.514$521,2010$.

[13] D. Jiang and L. Delgrossi, "IEEE 802.11p: Towards an international standard for wireless access in vehicular environments," IEEE Vehicular Technology Conference, 2008. VTC Spring 2008, pp.20362040, 2008. 
[14] K. Doppler, M. Rinne, C. Wijting, C. Ribeiro, and K. Hugl, "Deviceto-device communication as an underlay to lte-advanced networks," IEEE Commun. Mag., vol.47, no.12, pp.42-49, 2009.

[15] N. Golrezaei, A.F. Molisch, A.G. Dimakis, and G. Caire, "Femtocaching and device-to-device collaboration: A new architecture for wireless video distribution," IEEE Commun. Mag., vol.51, no.4, pp.142-149, 2013.

[16] T.L. Marzetta, "Noncooperative cellular wireless with unlimited numbers of base station antennas," IEEE Trans. Wireless Commun., vol.9, no.11, pp.3590-3600, 2010.

[17] F. Rusek, D. Persson, B.K. Lau, E.G. Larsson, T.L. Marzetta O. Edfors, and F. Tufvesson, "Scaling up MIMO: Opportunities and challenges with very large arrays," IEEE Signal Process. Mag., vol.30, no.1, pp.40-60, 2013.

[18] E.G. Larsson, F. Tufvesson, O. Edfors, and T.L. Marzetta, "Massive MIMO for next generation wireless systems," IEEE Commun. Mag. vol.52, no.2, pp.186-195, Feb. 2014.

[19] Y.-H. Nam, B.L. Ng, K. Sayana, Y. Li, J. Zhang, Y. Kim, and J. Lee, "Full-dimension MIMO (fd-MIMO) for next generation cellular technology," IEEE Commun. Mag., vol.51, no.6, pp.172-179, 2013.

[20] A.F. Molisch, Wireless communications, IEEE Press - Wiley, 2nd ed., 2010.

[21] A.F. Molisch, L.J. Greenstein, and M. Shafi, "Propagation issues for cognitive radio," Proc. IEEE, vol.97, no.5, pp.787-804, 2009.

[22] M. Steinbauer, A.F. Molisch, and E. Bonek, "The double-directional radio channel," IEEE Antennas Propag. Mag., vol.43, no.4, pp.5163, 2001.

[23] A.F. Molisch, A. Kuchar, J. Laurila, K. Hugl, and R. Schmalenberger, "Geometry-based directional model for mobile radio channels - Principles and implementation," European Transactions on Telecommunications, vol.14, no.4, pp.351-359, 2003.

[24] U. Martin, "Spatio-temporal radio channel characteristics in urban macrocells," IEE Proc. Radar, Sonar and Navigation, vol.145, no.1, pp.42-49, 1998.

[25] T. Zwick, C. Fischer, D. Didascalou, and W. Wiesbeck, "A stochastic spatial channel model based on wave-propagation modeling," IEEE J. Sel. Areas Commun., vol.18, no.1, pp.6-15, 2000.

[26] M. Toeltsch, J. Laurila, K. Kalliola, A.F. Molisch, P. Vainikainen, and E. Bonek, "Statistical characterization of urban spatial radio channels," IEEE J. Sel. Areas Commun., vol.20, no.3, pp.539-549, 2002.

[27] L. Vuokko, P. Vainikainen, and J.-I. Takada, "Clusterization of measured direction-of-arrival data in an urban macrocellular environment," 14th IEEE Proc. Personal, Indoor and Mobile Radio Communications, 2003. PIMRC 2003, vol.2, pp.1222-1226, 2003.

[28] N. Czink, P. Cera, J. Salo, E. Bonek, J.-P. Nuutinen, and J. Ylitalo, "A framework for automatic clustering of parametric MIMO channel data including path powers," 2006 IEEE 64th Vehicular Technology Conference, 2006. VTC-2006 Fall, pp.1-5, 2006.

[29] H. Asplund, A.F. Molisch, M. Steinbauer, and N. Mehta, "Clustering of scatterers in mobile radio channels-evaluation and modeling in the COST259 directional channel model," IEEE International Conference on Communications, 2002. ICC 2002, vol.2, pp.901-905, 2002.

[30] M. Zhu, F. Tufvesson, and J. Medbo, "Correlation properties of large scale parameters from $2.66 \mathrm{GHz}$ multi-site macro cell measurements," 2011 IEEE 73rd Vehicular Technology Conference (VTC Spring), pp.1-5, May 2011.

[31] B.K. Lau, M. Jensen, J. Medbo, and J. Furuskog, "Single and multiuser cooperative MIMO in a measured urban macrocellular environment," IEEE Trans. Antennas Propag., vol.60, pp.624-632, Feb. 2012.

[32] S. Jaeckel, L. Jiang, V. Jungnickel, L. Thiele, C. Jandura, G. Sommerkorn, and C. Schneider, "Correlation properties of large and small-scale parameters from multicell channel measurements," 3rd European Conference on Antennas and Propagation, 2009. Eu-
CAP 2009, pp.3406-3410, March 2009

[33] X. Yin, J. Liang, Y. Fu, Z. Zhang, J.-J. Park, M.-D. Kim, and H.K. Chung, "Measurement-based stochastic models for the crosscorrelation of multi-link small-scale fading in cooperative relay environments," 2012 6th European Conference on Antennas and Propagation (EUCAP), pp.1-5, 2012

[34] G. Dahman, J. Flordelis, and F. Tufvesson, "On the cross-correlation properties of large-scale fading in distributed antenna systems," IEEE Wireless Communications and Networking Conference, April 2014.

[35] C. Oestges, P. Castiglione, and N. Czink, "Empirical modeling of nomadic peer-to-peer networks in office environment," 2011 IEEE 73rd Vehicular Technology Conference (VTC Spring), pp.1-5, May 2011

[36] J. Poutanen, F. Tufvesson, K. Haneda, V. Kolmonen, and P. Vainikainen, "Multi-link MIMO channel modeling using geometry-based approach," IEEE Trans. Antennas Propag., vol.60, pp.587-596, Feb. 2012.

[37] L. Liu, C. Oestges, J. Poutanen, K. Haneda, P. Vainikainen, F. Quitin, F. Tufvesson, and P. Doncker, "The COST 2100 MIMO channel model," IEEE Wireless Commun., vol.19, pp.92-99, Dec. 2012.

[38] J. Karedal, N. Czink, A. Paier, F. Tufvesson, and A.F. Molisch, "Path loss modeling for vehicle-to-vehicle communications," IEEE Trans. Veh. Technol., vol.60, pp.323-328, Jan 2011.

[39] T. Mangel, O. Klemp, and H. Hartenstein, " $5.9 \mathrm{GHz}$ inter-vehicle communication at intersections: A validated non-line-of-sight pathloss and fading model," EURASIP Journal on Wireless Communications and Networking, vol.2011, no.1, p.182, 2011.

[40] T. Abbas, A. Thiel, T. Zemen, C.F. Mecklenbräuker, and F. Tufvesson, "Validation of a non-line-of-sight path-loss model for V2V communications at street intersections," 13th International Conference on ITS Telecommunications, Tampere, Finalnd, 2013$11-05,2013$

[41] Z. Li, R. Wang, and A.F. Molisch, "Shadowing in urban environments with microcellular or peer-to-peer links," 2012 6th European Conference on Antennas and Propagation (EUCAP), pp.44-48, March 2012.

[42] M. Schack, J. Nuckelt, R. Geise, L. Thiele, and T. Kurner, "Comparison of path loss measurements and predictions at urban crossroads for c2c communications," Proc. 5th European Conference on Antennas and Propagation (EUCAP), pp.2896-2900, April 2011.

[43] C. Mecklenbrauker, A.F. Molisch, J. Karedal, F. Tufvesson, A. Paier, L. Bernado, T. Zemen, O. Klemp, and N. Czink, "Vehicular channel characterization and its implications for wireless system design and performance," Proc. IEEE, vol.99, pp.1189-1212, July 2011.

[44] A.F. Molisch, F. Tufvesson, J. Karedal, and C. Mecklenbrauker, "A survey on vehicle-to-vehicle propagation channels," IEEE Wireless Commun., vol.16, pp.12-22, Dec. 2009.

[45] L. Bernado, T. Zemen, F. Tufvesson, A.F. Molisch, and C. Mecklenbrauker, "Delay and Doppler spreads of nonstationary vehicular channels for safety-relevant scenarios," IEEE Trans. Veh. Technol., vol.63, pp.82-93, Jan. 2014.

[46] O. Renaudin, Experimental channel characterization for vehicle-tovehicle communication systems. $\mathrm{PhD}$ thesis, Universite Catholique de Lovain, Belgium, 2013.

[47] L. Bernado, T. Zemen, F. Tufvesson, A.F. Molisch, and C. Mecklenbrauker, "The (in-) validity of the WSSUS assumption in vehicular radio channels," 2012 IEEE 23rd International Symposium on Personal Indoor and Mobile Radio Communications (PIMRC), pp.1757-1762, Sept. 2012

[48] J. Karedal, F. Tufvesson, N. Czink, A. Paier, C. Dumard, T. Zemen, C. Mecklenbrauker, and A.F. Molisch, "A geometry-based stochastic MIMO model for vehicle-to-vehicle communications," IEEE Trans. Wireless Commun., vol.8, pp.3646-3657, July 2009.

[49] M. Walter, D. Shutin, and U.-C. Fiebig, "Delay-dependent Doppler probability density functions for vehicle-to-vehicle scatter chan- 
nels," IEEE Trans. Antennas Propag., vol.62, no.4, pp.2238-2249, 2014.

[50] O. Renaudin, V. Kolmonen, P. Vainikainen, and C. Oestges, "Nonstationary narrowband MIMO inter-vehicle channel characterization in the 5-GHz band," IEEE Trans. Veh. Technol., vol.59, pp.20072015, May 2010

[51] G. Acosta-Marum and M. Ingram, "Six time- and frequency- selective empirical channel models for vehicular wireless LANs," IEEE Veh. Technol. Mag., vol.2, no.4, pp.4-11, 2007.

[52] J. Karedal, P. Almers, A. Johansson, F. Tufvesson, and A.F. Molisch, "A MIMO channel model for wireless personal area networks," IEEE Trans. Wireless Commun., vol.9, no.1, pp.245-255, 2010.

[53] A. Kammoun, H. Khanfir, Z. Altman, M. Debbah, and M. Kamoun, "Survey on 3D channel modeling: From theory to standardization," arXiv preprint arXiv:1312.0288, 2013

[54] X. Gao, F. Tufvesson, and O. Edfors, "Massive MIMO channels Measurements and models," 47th Annual Asilomar Conference on Signals, Systems, and Computers, Nov. 2013.

[55] J. Fuhl, J.-P. Rossi, and E. Bonek, "High-resolution 3-d direction-ofarrival determination for urban mobile radio," IEEE Trans. Antennas Propag., vol.45, pp.672-682, April 1997.

[56] A. Kuchar, J.-P. Rossi, and E. Bonek, "Directional macro-cell channel characterization from urban measurements," IEEE Trans. Antennas Propag., vol.48, no.2, pp.137-146, 2000

[57] K. Kalliola, K. Sulonen, H. Laitinen, O. Kivekas, J. Krogerus, and P. Vainikainen, "Angular power distribution and mean effective gain of mobile antenna in different propagation environments," IEEE Trans. Veh. Technol., vol.51, pp.823-838, Sept. 2002.

[58] C. Schneider, M. Narandzic, M. Kaske, G. Sommerkorn, and R. Thoma, "Large scale parameter for the WINNER II channel model at $2.53 \mathrm{GHz}$ in urban macro cell," 2010 IEEE 71st Vehicular Technology Conference (VTC 2010-Spring), pp.1-5, May 2010.

[59] F. Quitin, C. Oestges, F. Horlin, and P. De Doncker, "A polarized clustered channel model for indoor multiantenna systems at 3.6 GHz," IEEE Trans. Veh. Technol., vol.59, no.8, pp.3685-3693, 2010

[60] J. Laurila, K. Kalliola, M. Toeltsch, K. Hugl, P. Vainikainen, and E. Bonek, "Wideband 3D characterization of mobile radio channels in urban environment," IEEE Trans. Antennas Propag., vol.50, pp.233-243, Feb. 2002.

[61] M. Toeltsch, J. Laurila, K. Kalliola, A.F. Molisch, P. Vainikainen, and E. Bonek, "Statistical characterization of urban spatial radio channels," IEEE J. Sel. Areas Commun., vol.20, pp.539-549, April 2002

[62] J. Medbo, H. Asplund, J.E. Berg, and N. Jalden, "Directional channel characteristics in elevation and azimuth at an urban macrocel base station," 2012 6th European Conference on Antennas and Propagation (EUCAP), pp.428-432, 2012.

[63] F. Pei, J. Zhang, and C. Pan, "Elevation angle characteristics of urban wireless propagation environment at $3.5 \mathrm{GHz}, 2013$ IEEE 78th Vehicular Technology Conference (VTC Fall), pp.1-5, Sept. 2013.

[64] T.A. Thomas, F.W. Vook, E. Mellios, G.S. Hilton, A.R. Nix, and E. Visotsky, "3D extension of the 3GPP/ITU channel model," 2013 IEEE 77th Vehicular Technology Conference (VTC Spring), pp.1-5, June 2013

[65] K. Kitao, T. Imai, K. Saito, and Y. Okumura, "Elevation directional channel properties at bs to evaluate 3D beamforming," 2013 International Symposium on Intelligent Signal Processing and Communications Systems (ISPACS), pp.640-644, 2013.

[66] L. Hentila, P. Kyosti, and J. Meinila, "Elevation extension for a geometry-based radio channel model and its influence on MIMO antenna correlation and gain imbalance," Proc. 5th European Conference on Antennas and Propagation (EUCAP), pp.2175-2179, April 2011.

[67] C. Huang, J. Zhang, X. Nie, and Y. Zhang, "Cluster characteristics of wideband MIMO channel in indoor hotspot scenario at $2.35 \mathrm{GHz}$," 2009 IEEE 70th Vehicular Technology Conference Fall (VTC 2009-
Fall), pp.1-5, Sept. 2009

[68] R. Wang, S. Sangodoyin, A.F. Molisch, J. Zhang, Y.H. Nam, and J. Lee, "Elevation characteristics of outdoor-to-indoor macrocellular propagation channels," Proc. VTC 2014 spring, 2014.

[69] T. Rappaport, S. Sun, R. Mayzus, H. Zhao, Y. Azar, K. Wang, G. Wong, J. Schulz, M. Samimi, and F. Gutierrez, "Millimeter wave mobile communications for 5G cellular: It will work!," IEEE Access, vol.1, pp.335-349, 2013.

[70] F. Giannetti, M. Luise, and R. Reggiannini, "Mobile and personal communications in the $60 \mathrm{GHz}$ band: A survey," Wireless Pers. Commun., vol.10, no.2, pp.207-243, 1999.

[71] Z. Qingling and J. Li, "Rain attenuation in millimeter wave ranges," ISAPE'06. 7th International Symposium on Antennas, Propagation \& EM Theory, 2006, pp.1-4, 2006.

[72] H. Xu, T. Rappaport, R. Boyle, and J. Schaffner, "Measurements and models for 38-GHz point-to-multipoint radiowave propagation," IEEE J. Sel. Areas Commun., vol.18, pp.310-321, March 2000.

[73] H. Xu, T. Rappaport, R. Boyle, and J. Schaffner, "38-GHz wideband point-to-multipoint measurements under different weather conditions," IEEE Commun. Lett., vol.4, pp.7-8, Jan. 2000.

[74] A. Kajiwara, "LMDS radio channel obstructed by foliage," 2000 IEEE International Conference on Communications, 2000. ICC 2000, vol.3, pp.1583-1587, 2000.

[75] D.L. Jones, R.H. Espeland, and E.J. Violette, Vegetation loss measurements at 9.6, 28.8, 57.6, and $96.1 \mathrm{GHz}$ through a conifer orchard in Washington state. US Department of Commerce, National Telecommunications and Information Administration, 1989.

[76] T. Rappaport, F. Gutierrez, E. Ben-Dor, J. Murdock, Y. Qiao, and J. Tamir, "Broadband millimeter-wave propagation measurements and models using adaptive-beam antennas for outdoor urban cellular communications," IEEE Trans. Antennas Propag., vol.61, pp.18501859, April 2013.

[77] C. Gustafson and F. Tufvesson, "Characterization of $60 \mathrm{GHz}$ shadowing by human bodies and simple phantoms," 2012 6th European Conference on Antennas and Propagation (EUCAP), pp.473-477, March 2012.

[78] P. Smulders and A. Wagemans, "Wideband indoor radio propagation measurements at $58 \mathrm{GHz}$," Electron. Lett., vol.28, pp.1270-1272, June 1992.

[79] N. Daniele, D. Chagnot, and C. Fort, "Outdoor millimetre-wave propagation measurements with line of sight obstructed by natural elements," Electron. Lett., vol.30, pp.1533-1534, Sept. 1994.

[80] Y. Azar, G. Wong, K. Wang, R. Mayzus, J. Schulz, H. Zhao, F. Gutierrez, D. Hwang, and T. Rappaport, " $28 \mathrm{GHz}$ propagation measurements for outdoor cellular communications using steerable beam antennas in New York city," 2013 IEEE International Conference on Communications (ICC), pp.5143-5147, June 2013.

[81] M. Samimi, K. Wang, Y. Azar, G.N. Wong, R. Mayzus, H. Zhao, J.K. Schulz, S. Sun, F. Gutierrez, and T.S. Rappaport, " $28 \mathrm{GHz}$ angle of arrival and angle of departure analysis for outdoor cellular communications using steerable beam antennas in New York City," 2013 IEEE 77th Vehicular Technology Conference (VTC Spring), pp.1-6, June 2013.

[82] S. Yong, et al., " $\mathrm{Tg} 3 \mathrm{c}$ channel modeling sub-committee final report," IEEE802. 15-07-0584-00-003c, 2007.

[83] T. Baykas, C.-S. Sum, Z. Lan, J. Wang, M. Rahman, H. Harada, and S. Kato, "IEEE 802.15.3c: the first IEEE wireless standard for data rates over $1 \mathrm{~Gb} / \mathrm{s}$," IEEE Commun. Mag., vol.49, pp.114-121, July 2011.

[84] A. Maltsev, et al., "Channel models for $60 \mathrm{GHz}$ WLAN systems," IEEE802.11-09-0334r8, 2010.

[85] C. Gustafson, K. Haneda, S. Wyne, and F. Tufvesson, "On mm-wave multipath clustering and channel modeling," IEEE Trans. Antennas Propag., vol.62, pp.1445-1455, March 2014.

[86] C. Gustafson, F. Tufvesson, S. Wyne, K. Haneda, and A.F. Molisch, "Directional analysis of measured $60 \mathrm{GHz}$ indoor radio channels using SAGE," 2011 IEEE 73rd Vehicular Technology Conference 
(VTC Spring), pp.1-5, May 2011.

[87] A. Adhikary, E.A. Safadi, M. Samimi, R. Wang, G. Caire, T.S. Rappaport, and A.F. Molisch, "Joint spatial division and multiplexing for mm-wave channels," IEEE J. Sel. Areas Commun., vol.32, pp.1239-1255, 2014

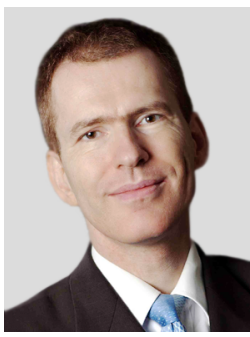

Andreas F. Molisch received the Dipl. Ing., Ph.D., and habilitation degrees from the Technical University of Vienna, Austria, in 1990, 1994, and 1999, respectively. He subsequently was with AT\&T (Bell) Laboratories Research (USA); Lund University, Sweden, and Mitsubishi Electric Research Labs (USA). He is now a Professor of Electrical Engineering with the University of Southern California, Los Angeles. His current research interests are the measurement and modeling of mobile radio channels, ultra-wideband communications and localization, cooperative communications, multiple-input-multiple-output systems, wireless systems for healthcare, and novel cellular architectures. He has authored, coauthored, or edited four books (among them the textbook Wireless Communications, Wiley-IEEE Press), 16 book chapters, some 160 journal papers, and numerous conference contributions, as well as more than 70 patents and 60 standards contributions. Dr. Molisch has been an Editor of a number of journals and special issues, General Chair, Tecnical Program Committee Chair, or Symposium Chair of multiple international conferences, as well as Chairman of various international standardization groups. He is a Fellow of the IEEE, Fellow of the AAAS, Fellow of the IET, an IEEE Distinguished Lecturer, a member of the Austrian Academy of Sciences, and recipient of numerous awards.

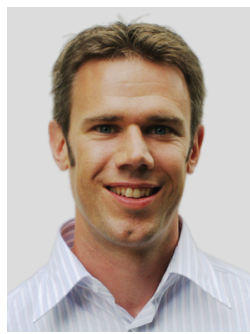

Fredrik Tufvesson received his Ph.D. in 2000 from Lund University in Sweden. After two years at a startup company, he joined the department of Electrical and Information Technology at Lund University, where he is now professor in radio systems. His main research interests are channel modeling and measurements for wireless communication, including channels for both MIMO and UWB systems. Beside this, he also works on Massive MIMO, distributed antenna systems and radio based positioning. 